\title{
Optical tomographic imaging for breast cancer detection
}

Wenxiang Cong

Xavier Intes

Ge Wang 


\title{
Optical tomographic imaging for breast cancer detection
}

\author{
Wenxiang Cong, ${ }^{*}$ Xavier Intes, and Ge Wang \\ Rensselaer Polytechnic Institute, Biomedical Imaging Center, Department of Biomedical Engineering, Troy, New York, United States
}

\begin{abstract}
Diffuse optical breast imaging utilizes near-infrared (NIR) light propagation through tissues to assess the optical properties of tissues for the identification of abnormal tissue. This optical imaging approach is sensitive, cost-effective, and does not involve any ionizing radiation. However, the image reconstruction of diffuse optical tomography (DOT) is a nonlinear inverse problem and suffers from severe illposedness due to data noise, NIR light scattering, and measurement incompleteness. An image reconstruction method is proposed for the detection of breast cancer. This method splits the image reconstruction problem into the localization of abnormal tissues and quantification of absorption variations. The localization of abnormal tissues is performed based on a well-posed optimization model, which can be solved via a differential evolution optimization method to achieve a stable reconstruction. The quantification of abnormal absorption is then determined in localized regions of relatively small extents, in which a potential tumor might be. Consequently, the number of unknown absorption variables can be greatly reduced to overcome the underdetermined nature of DOT. Numerical simulation experiments are performed to verify merits of the proposed method, and the results show that the image reconstruction method is stable and accurate for the identification of abnormal tissues, and robust against the measurement noise of data. ๑ 2017 Society of Photo-Optical Instrumentation Engineers (SPIE) [DOI: 10.1117/1.JBO.22.9.096011]
\end{abstract}

Keywords: diffuse optical tomography; breast imaging; image reconstruction; differential evolution; compressive sensing. Paper 170248RR received Apr. 18, 2017; accepted for publication Aug. 21, 2017; published online Sep. $20,2017$.

\section{Introduction}

Breast cancer is a major health problem. If this disease is treated at an early stage using current therapies, patient prognosis improves significantly. Currently, multiple methods are available for breast cancer screening and diagnosis. The x-ray mammography is an effective screening technique. However, the breast consists of tissues with similar densities and has similar attenuation coefficients. The appearance of cancer on mammograms has a substantial similarity to that of normal tissues. The $\mathrm{X}$-ray mammography is less sensitive in dense breast. ${ }^{1} \mathrm{X}$-rays are also a type of ionizing radiation that bears a risk of cancer induction. Magnetic resonance imaging (MRI) is another effective and sensitive technique to detect cancer in dense tissues. ${ }^{2}$ It has a very high negative predictive value, which helps to distinguish benign tumors and malignant tumors, and decrease the possibility of false-negative diagnosis. A major disadvantage of MRI is that it is much more expensive and time-consuming than other breast-diagnostic methods. Ultrasonography can delineate cysts, and benign and malignant masses. But it is limited by poor soft-tissue contrast, inherent speckle noise, and strong operator dependence. ${ }^{3}$ The optical molecular imaging modality uses exogenous fluorescent probes as additional contrast agents that target molecules relevant to breast cancer. ${ }^{4}$ The use of fluorescent probes has a potential in early breast cancer detection but the effectiveness of the fluorescence imaging relies on the functions of the probes. ${ }^{5}$ Molecular contrast probes on specific tumor receptors or tumor-associated enzymes are also under development.

Diffuse optical tomography (DOT) was introduced in the early 1990s. DOT uses near-infrared (NIR) light transmission and intrinsic breast tissue contrast for the detection and characterization of abnormal tissue. ${ }^{6}$ It is sensitive, cost-effective, does not involve any ionizing radiation, and has a high sensitivity due to the rich optical absorption contrast. Owing to the relatively low absorption of hemoglobin, water, and lipid at wavelengths of 650 to $1000 \mathrm{~nm}$, NIR light can transmit through several centimeters of biological tissue with an adequate signal-to-noise ratio for breast tomographic imaging. ${ }^{7}$ Furthermore, in this spectral range, oxyhemoglobin, deoxyhemoglobin, and lipids predominantly affect the absorptive properties of the breast tissue. There is a difference in total hemoglobin concentration levels between benign and malignant breast lesions, so it was a useful indicator for distinguishing between benign and malignant breast lesions. ${ }^{8}$ By combining images reconstructed by DOT at various wavelengths, concentrations of oxy- and deoxy hemoglobins and water can be determined to reveal tumors from background tissue. ${ }^{9}$

However, NIR light is strongly scattering in biological tissues, the measurable quantity can only be collected on a partial external surface of an object, and light fields obtained from different excitation sources are highly correlated. The resultant volumetric reconstruction is a nonlinear inverse problem and suffers from severe illposedness exaggerated by data noise, NIR light scattering, and measurement incompleteness. The image reconstruction is still the most challenging imaging tasks. Iterative methods and linearization methods are popular image reconstruction algorithms for the optical tomography. ${ }^{10,11}$ The regularization or stabilization techniques are often applied to obtain physically realistic results, such as Tikhonov regularization, and $l_{1}$ - and $l_{p}$-norm regularizations. ${ }^{12-14}$ However, these methods cannot 
fundamentally solve the illposed problem of the DOT, usually resulting in aberrant image reconstruction in the presence of measurement noise.

In this paper, we propose a new diffuse optical tomographic imaging method for the detection of breast cancer. This method divides the image reconstruction problem into the localization of abnormal tissues and the quantification of absorption variations. The localization of abnormal tissues is performed based on a well-posed optimization model, which can be solved using differential evolution (DE) global optimization method. The quantification of abnormal absorption variations is then determined in localized regions of relatively small extents, in which potentially tumors might be. This method is robust against the measurement noise of data and produces accurate and stable image reconstruction of abnormal absorption variations in the breast.

\section{Image Reconstruction}

\subsection{Physical Model}

Optical imaging utilizes light propagating through tissue for the assessment of optical properties. The propagation of light in biological tissues is a complex process involving both absorption and scattering of light. The light propagation model describes the interaction of photons with tissues, and predicts a light intensity distribution in the breast and on its external surface. By minimizing the discrepancy between the predicted data and measured data, an image reconstruction algorithm can recover the spatial distribution of intrinsic optical tissue properties. The radiative transfer equation (RTE) accurately models the photon propagation in biological tissues. ${ }^{15,16}$ Due to the extensive computational cost, it is difficult to directly apply RTE for biomedical tomographic imaging. The diffusion approximation (DA) model to RTE is widely used to describe the NIR light transport in biological tissues with a high computational efficiency and an adequate accuracy in strongly scattering and weakly absorbing objects. ${ }^{15}$ The steady-state DA model is expressed as follows:

$$
-\nabla \cdot[D(\mathbf{r}) \nabla \Phi(\mathbf{r})]+\mu_{a}(\mathbf{r}) \Phi(\mathbf{r})=S(\mathbf{r}), \quad \mathbf{r} \in \Omega,
$$

where $\mathbf{r}$ is a positional vector, $\Phi(\mathbf{r})$ is the NIR photon fluence rate, $S(\mathbf{r})$ is the NIR source, $\mu_{a}(\mathbf{r})$ is the absorption coefficient, $D(\mathbf{r})$ is the diffusion coefficient defined by $D(\mathbf{r})=$ $\left\{3\left[\mu_{a}(\mathbf{r})+\mu_{s}^{\prime}(\mathbf{r})\right]\right\}, \mu_{s}^{\prime}(\mathbf{r})$ is the reduced scattering coefficient, and $\Omega \subset R^{3}$ is a region of interest in the object. If no photon travels across the boundary $\partial \Omega$ into the tissue domain $\Omega$, DA is constrained by the Robin boundary condition ${ }^{17}$

$\Phi(\mathbf{r})+2 \alpha D(\mathbf{r})[v \cdot \nabla \Phi(\mathbf{r})]=0, \quad \mathbf{r} \in \partial \Omega$,

where $v$ is an outward unit normal vector on $\partial \Omega$, and $\alpha$ is the boundary mismatch factor between the tissue with a refractive index $n$ and air, which can be approximated by $\alpha=(1+\gamma) /(1-\gamma)$ with $\gamma=-1.44 n^{-2}+0.71 n^{-1}+0.67$ $+0.06 n{ }^{17}$ The measurable photon fluence on the surface of the object can be expressed as

$m(\mathbf{r})=-D(\mathbf{r})[\boldsymbol{\nu} \cdot \nabla \Phi(\mathbf{r})], \quad \mathbf{r} \in \partial \Omega$.

Breast tissue consists mainly of light elements with low atomic numbers, and its elemental composition is nearly uniform with little density variation. Because the scattering coefficient provides information about internal structure, the spatial variation of scattering coefficients in breast tissues is slow. ${ }^{12}$
The absorption coefficient is related to tissue chromophores (e.g., water, lipid, oxy- and deoxyhemoglobins), and the absorption imaging of hemoglobin is potentially useful in assessing tissue status and malignancy. Hence, in this context, we only focus on the absorption contrast since angiogenesis in cancer mainly causes a change in absorption properties of hemoglobin. Accordingly, we can decompose the absorption coefficients into $\mu_{a}(\mathbf{r})=\mu_{a}^{0}(\mathbf{r})+\delta \mu_{a}(\mathbf{r})$, where $\mu_{a}^{0}(\mathbf{r})$ is the absorption coefficient of normal breast tissue, and $\delta \mu_{a}(\mathbf{r})$ denotes the abnormal absorption variations in tissues, since malignant tissues show higher levels of absorption compared to healthy tissues. Hence, we can rewrite Eq. (1) as follows:

$$
-\nabla \cdot[D(\mathbf{r}) \nabla \Phi(\mathbf{r})]+\mu_{a}^{0}(\mathbf{r}) \Phi(\mathbf{r})+\delta \mu_{a}(\mathbf{r}) \Phi(\mathbf{r})=S(\mathbf{r}), \quad \mathbf{r} \in \Omega
$$

Equation (4) is the governing imaging model for the image reconstruction of abnormal tissues.

\subsection{Localization of Abnormal Absorptions}

The geometrical shape of the compressive breast can be accurately established via laser scanning. ${ }^{18}$ From the geometrical shape of the compressive breast, a finite-element mesh is generated using the computer graphic techniques (Amira software). Key reference points are marked on the breast. Accordingly, the markers also appear on the measured photon density image. Thus, the one-to-one relationship between all pixels on the measured photon density image and the coordinates of the finite-elements model can be established for the image registration. Based on the finite-element model, differential Eqs. (2) and (4) can be discretized into a matrix equation with respect to the photon fluence rate $\Phi(\mathbf{r})^{19}$

$\mathbf{A} \boldsymbol{\Phi}+\mathbf{B}(\boldsymbol{\delta}) \boldsymbol{\Phi}=\mathbf{S}$

where $\boldsymbol{\delta}=\left\{\delta_{1}, \delta_{2}, \cdots, \delta_{N}\right\}$ is a vector defining abnormal absorption variations at nodes $\delta \mu_{a}(\mathbf{r})$, the components of the matrix $\mathbf{A}$ is computed as

$$
\begin{aligned}
a_{i j}= & \int_{\Omega} D(\mathbf{r}) \nabla \varphi_{i}(\mathbf{r}) \cdot \nabla \varphi_{j}(\mathbf{r}) \mathrm{d} \mathbf{r}+\int_{\Omega} \mu_{a}^{0}(\mathbf{r}) \varphi_{i}(\mathbf{r}) \varphi_{j}(\mathbf{r}) \mathrm{d} \mathbf{r} \\
& +\int_{\partial \Omega} \varphi_{i}(\mathbf{r}) \varphi_{j}(\mathbf{r}) / 2 \alpha \mathrm{d} \mathbf{r}, \quad i, j=1,2, \cdots, N
\end{aligned}
$$

and the components of the matrix $\mathbf{B}$ is computed as

$$
b_{i j}=\sum_{k=1}^{N} \delta_{k} \int_{\Omega} \varphi_{k}(\mathbf{r}) \varphi_{i}(\mathbf{r}) \varphi_{j}(\mathbf{r}) \mathrm{d} \mathbf{r}, \quad i, j=1,2, \cdots, N
$$

where $\varphi_{i}(\mathbf{r})(i=1,2, \cdots, N)$ are the shape functions. Since the matrices $\mathbf{A}$ and $\mathbf{B}$ in Eq. (5) are positive definite, we have

$\boldsymbol{\Phi}=[\mathbf{A}+\mathbf{B}(\boldsymbol{\delta})]^{-1} \mathbf{S}$.

To simplify the computational complexity, Eq. (8) can be linearized to establish the linear relationship between the measured photon fluence rate $\boldsymbol{\Phi}(\Gamma)$ and abnormal absorption variations $\boldsymbol{\delta}$

$$
\boldsymbol{\Phi}_{\mathbf{0}}(\Gamma)-\boldsymbol{\Phi}(\Gamma)=\mathbf{A}^{-1}(\Gamma) \mathbf{B}\left(\boldsymbol{\Phi}_{\mathbf{0}}\right) \boldsymbol{\delta},
$$


where $\Gamma$ is a boundary nodal set on the finite-element mesh corresponding to the measurable photon fluence rates on upper surface and lower surface of the breast, $\boldsymbol{\Phi}_{0}=\mathbf{A}^{-1} \mathbf{S}$ is the photon fluence rate in case of absence of abnormal absorptions. To find a stable solution of Eq. (9), we introduce the following optimization model:

$$
\left\{\begin{array}{l}
{\left[\delta_{i_{k}}^{*}, p_{k}^{*}\right]=\arg \min \left\|\mathbf{A}^{-1}(\Gamma) \mathbf{B}\left(\mathbf{\Phi}_{\mathbf{0}}\right) \boldsymbol{\delta}-\left[\mathbf{\Phi}_{\mathbf{0}}(\Gamma)-\boldsymbol{\Phi}(\Gamma)\right]\right\|} \\
\delta_{i_{k}}>0, \quad k=1,2, \ldots, T \\
\delta_{j}=0, \quad j \notin\left\{i_{1}, i_{2}, \ldots, i_{T}\right\}
\end{array}\right.
$$

where the vector $\boldsymbol{\delta}$ includes $T$ positive nonzero values $\left\{\delta_{i_{1}}, \delta_{i_{2}}, \cdots, \delta_{i_{T}}\right\}$, which represents the abnormal absorption variations at some isolated locations, which are determined by the corresponding nodal indices $\left\{i_{1}, i_{2}, \cdots, i_{T}\right\}$, and $T$ is a known positive integer that can be estimated as prior knowledge. For breast imaging, $T$ represents the number of tumors in the breast. In general, it is reasonable to assume that $T$ is less than 3 at an early stage of breast tumor, allowing a relatively easier solution to the optimization model Eq. (10). Each nodal index $i_{k}(k=1,2, \cdots, T)$ corresponds to a tumor position in the breast, which is an approximation to the average of coordinates weighted by the absorption values. The absorption variation $\delta_{i_{k}}(k=1,2, \cdots, T)$ is also an important feature of the tumor.

Equation (10) represents an NP (nondeterministic polynomial time) problem and cannot be efficiently solved using a gradient-based optimization method. Hence, a global optimization method, DE, can be used to solve the optimization problem Eq. (10). DE regenerates a population through executing mutation, crossover, and selection. It is a powerful stochastic optimization strategy and has a superior converging behavior at a high precision. Especially, for the small number of variables, the DE method is very manageable. In case of the unknown $T$, we can heuristically determine the number of tumors by gradually increasing the number of $T$, and solving Eq. (10). For each reconstructed image, if two or more positive abnormal absorption variations are so close that they cannot be isolated within a user-specified spatial resolution, they should be combined into a single abnormality for the determination of tumor number.

\subsection{Quantification of Abnormal Absorption Variations}

Based on locations of tumors estimated by the reconstruction algorithm in above section, we can further quantify the intensities of abnormal absorption variations for tumors. We set a subregion $\Omega_{k}(k=1,2, \cdots, T)$ around the tumor position with a radius related to the estimation of the abnormal absorption variations $\delta_{i_{k}}^{*}(k=1,2, \cdots, T)$ to form a feasible region $\bar{\Omega}=\bigcup_{k=1}^{T} \Omega_{k}$ of abnormal absorption variations for image reconstruction. Region $\bar{\Omega}$ contains relatively small extents comparing with global region $\Omega$. Consequently, the number of unknown variables can be significantly reduced to overcome the underdetermined nature of DOT. From the feasible region $\bar{\Omega}$, we effectively perform image reconstruction using an algorithm for sparse linear equations and least squares ${ }^{19,20}$ based on Eq. (10) as follows:

$$
\left\{\begin{array}{l}
\boldsymbol{\delta}^{*}=\arg \min \left\|\mathbf{A}^{-1}(\Gamma) \mathbf{B}\left(\boldsymbol{\Phi}_{\mathbf{0}}\right) \boldsymbol{\delta}-\left[\boldsymbol{\Phi}_{\mathbf{0}}(\Gamma)-\boldsymbol{\Phi}(\Gamma)\right]\right\| \\
\boldsymbol{\delta} \in \bar{\Omega}
\end{array} .\right.
$$

\section{Numerical Simulations}

To quantify the performance of proposed image reconstruction method, a digital breast phantom is applied for the numerical experiments. The breast phantom was developed from clinical dual-energy x-ray CT. ${ }^{21,22}$ The phantom was defined from the tetrahedral mesh with 65,196 tetrahedral elements and 14,490 nodes, as shown in Fig. 1. The fibroglandular tissue volume fraction map $C_{f}(r)$ is obtained from digital mammogram. In the numerical simulations, it is assumed that the primary breast tissue constituents are fibroglandular and adipose tissue, and the optical properties can be estimated by

$\mu(r)=C_{f}(r) \mu_{\mathrm{fib}}+C_{a}(r) \mu_{\mathrm{adi}}$

where $C_{a}(r)$ is the adipose tissue volume fraction at location $r$, which can be computed by $1-C_{f}(r)$.

The absorption coefficients $\mu_{\text {adi }}$ and $\mu_{\text {fib }}$ at wavelength $\lambda$ can be calculated as follows:

$$
\begin{aligned}
& \mu_{\mathrm{fib}}(\lambda)=\ln (10) \varepsilon_{\mathrm{HbO}_{2}}(\lambda) C_{\mathrm{HbO}_{2}}(\text { fib })+\ln (10) \varepsilon_{\mathrm{Hb}}(\lambda) C_{\mathrm{Hb}}(\text { fib }), \\
& \mu_{\text {adi }}(\lambda)=\ln (10) \varepsilon_{\mathrm{HbO}_{2}}(\lambda) C_{\mathrm{HbO}_{2}}(\text { adi })+\ln (10) \varepsilon_{\mathrm{Hb}}(\lambda) C_{\mathrm{Hb}}(\text { adi })
\end{aligned}
$$

where $C_{\mathrm{HbO}_{2}}$ is the oxyhemoglobin concentration $(\mu \mathrm{M}), C_{\mathrm{Hb}}$ is the deoxyhemoglobin concentration $(\mu \mathrm{M}), \varepsilon_{\mathrm{HbO}_{2}}(\lambda)$ and $\varepsilon_{\mathrm{Hb}}(\lambda)$ are the molar extinction coefficients of oxyhemoglobin $\left(\mathrm{HbO}_{2}\right)$ and deoxyhemoglobin $(\mathrm{Hb})$, respectively, and $\varepsilon_{\mathrm{HbO}_{2}}(\lambda)=$ $97.4\left(\mathrm{~mm}^{-1} \mathrm{M}^{-1}\right)$ and $\varepsilon_{\mathrm{Hb}}(\lambda)=69.304\left(\mathrm{~mm}^{-1} \mathrm{M}^{-1}\right)$ for $\lambda=830 \mathrm{~nm} .{ }^{23}$ Hence, the optical coefficients (absorption and reduced scattering coefficients) $\mu_{\text {adi }}$ and $\mu_{\text {fib }}$ are, respectively, determined from optical properties of adipose and fibroglandular based on the data in Table $1 .{ }^{23-25}$ The breast phantom included two tumors (denoted as tumor I and tumor II, as shown in Fig. 1) in a three-dimensional (3-D) Gaussian shape expressed as $S(\mathbf{r})=\exp \left(-\left\|\mathbf{r}-\mathbf{r}_{0}\right\|^{2} / 2 \sigma^{2}\right)$, where $\mathbf{r}_{\mathbf{0}}$ is the centroid of the tumor, and $\sigma$ is the standard deviation of the Gaussian sphere. The optical parameters in the tumors are given by

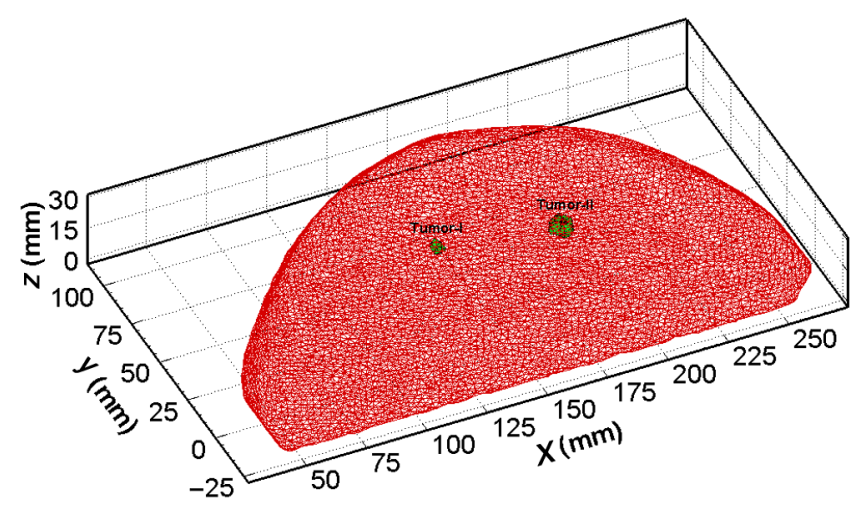

Fig. 1 3-D finite-element model of the breast phantom. 
Table 1 Optical parameters.

\begin{tabular}{lccc} 
Tissue type & $C_{\mathrm{HbO}_{2}}(\mu \mathrm{M})$ & $\mathrm{C}_{\mathrm{Hb}}(\mu \mathrm{M})$ & $\begin{array}{l}\mu_{s}^{\prime}\left(\mathrm{mm}^{-1}\right) \\
\text { at } 830 \mathrm{~nm}\end{array}$ \\
\hline Adipose (adi) & 13.84 & 4.81 & 0.713 \\
Fibroglandular (fib) & 18.96 & 6.47 & 0.775 \\
Malignant (mal) & 20.60 & 6.72 & 0.801 \\
\hline
\end{tabular}

$$
\begin{aligned}
\mu(r)= & {\left[1-C_{t}(r)\right]\left[C_{f}(r) \mu_{\mathrm{fib}}+C_{a}(r) \mu_{\mathrm{adi}}\right] } \\
& +C_{t}(r)\left[\mu_{\mathrm{adi}}+\gamma\left(\mu_{\mathrm{mal}}-\mu_{\mathrm{adi}}\right)\right]
\end{aligned}
$$

where $C_{t}(r)$ is the tumor volume fraction, $\gamma$ is a parameter to control the optical tumor contrast, ${ }^{21}$ and $\mu_{\text {mal }}$ is the optical coefficient for a malignant tumor calculated from the data in Table 1 using

$$
\begin{aligned}
\mu_{\mathrm{mal}}(\lambda)= & \ln (10) \varepsilon_{\mathrm{HbO}_{2}}(\lambda) C_{\mathrm{HbO}_{2}}(\mathrm{mal}) \\
& +\ln (10) \varepsilon_{\mathrm{Hb}}(\lambda) C_{\mathrm{Hb}}(\mathrm{mal})
\end{aligned}
$$

We assume that the NIR light source operated in a continuous wave $(\mathrm{CW})$ mode at $830 \mathrm{~nm}$. The optical signals were collected with 2622 detectors on the upper surface and 2473 detectors on the lower surface of the breast phantom. In the numerical experiments with the breast phantom, we set the centroid of tumor I at $(140,49,15) \mathrm{mm}$ and the centroid of tumor II at $(188,41$, $15) \mathrm{mm}$ in the finite-element coordinate system. To simulate different absorption contrasts, we defined $\gamma=1.2$ for tumor I to simulate a low-contrast tumor, and $\gamma=2$ for tumor II to simulate high-contrast tumor. The standard deviations of Gaussian tumors were assigned as $\sigma=2.3$ for tumor I and $\sigma=3.6$ for tumor II, respectively. Based on these parametric settings, the diffusion equation-based forward model was numerically solved using our in-house finite-element solver to generate CW optical simulation data. Then, we respectively added 30- and 25-dB Gaussian noise to generate more realistic datasets.

The image reconstruction algorithm described in Sec. 2 was applied to identify the abnormal absorption variations from the simulated datasets. First, the optimization model Eq. (10) was performed to estimate the positions of tumors. From the dataset with 30-dB noise level, tumor I and tumor II locations were estimated at $(140.18,47.60,15.27) \mathrm{mm}$ and $(188.33,40.52$, 14.32) $\mathrm{mm}$, respectively. From the dataset with $25-\mathrm{dB}$ noise level, tumor I and tumor II locations were identified at (142.38, 48.92, 15.24) $\mathrm{mm}$ and $(190.71,41.40,14.02) \mathrm{mm}$, respectively. Furthermore, we established a feasible region $\bar{\Omega}$ around every determined tumor location. Theoretically, the greater the absorption intensity value, the larger region the tumor occupies. From $\delta_{k}^{*}(k=1,2)$ obtained from Eq. (10), we can roughly estimate sizes of tumor I and tumor II, respectively. In fact, an approximate formula $D_{k}=e \cdot \delta_{k}^{*} /\left(\mu_{m}-\mu_{0}\right)(k=1,2)$ was derived from the BeerLambert law to estimate tumor sizes, where $\mu_{m}$ is the approximate absorption coefficient of a malignant tumor, ${ }^{26} \mu_{0}$ is the approximate absorption coefficient of the normal tissue, and $e$ is an approximate average size of finite elements. Then, spherical regions $R_{k}(k=1,2)$ of radius $D_{k}(k=1,2)$ can be established around the determined tumor locations. Hence, we obtain $\bar{\Omega}=R_{1} \cup R_{2}$. The $\bar{\Omega}$ is of a relatively small extent, and the number of unknown variables can be greatly reduced to overcome the underdetermined nature of DOT. In practical computation, due to the discretization and computational errors, the radius $D_{k}$ may be properly increased to ensure tumor regions in $\bar{\Omega}$. In our simulation, the selected radius was $D_{1}=7 \mathrm{~mm}$ for tumor I and $D_{2}=8 \mathrm{~mm}$ for tumor II. The optimization model Eq. (11) was implemented to quantify abnormal absorption variations from two datasets with $30-$ and $25-\mathrm{dB}$ noise levels, respectively. Figures 2 and 3 show the reconstructed abnormal absorption and variations from the simulation dataset with 30 and $25 \mathrm{~dB}$ noise levels, respectively. Table 2 presented the quantification results of image reconstruction. To compare with the state-of-the-art method, we also conducted the
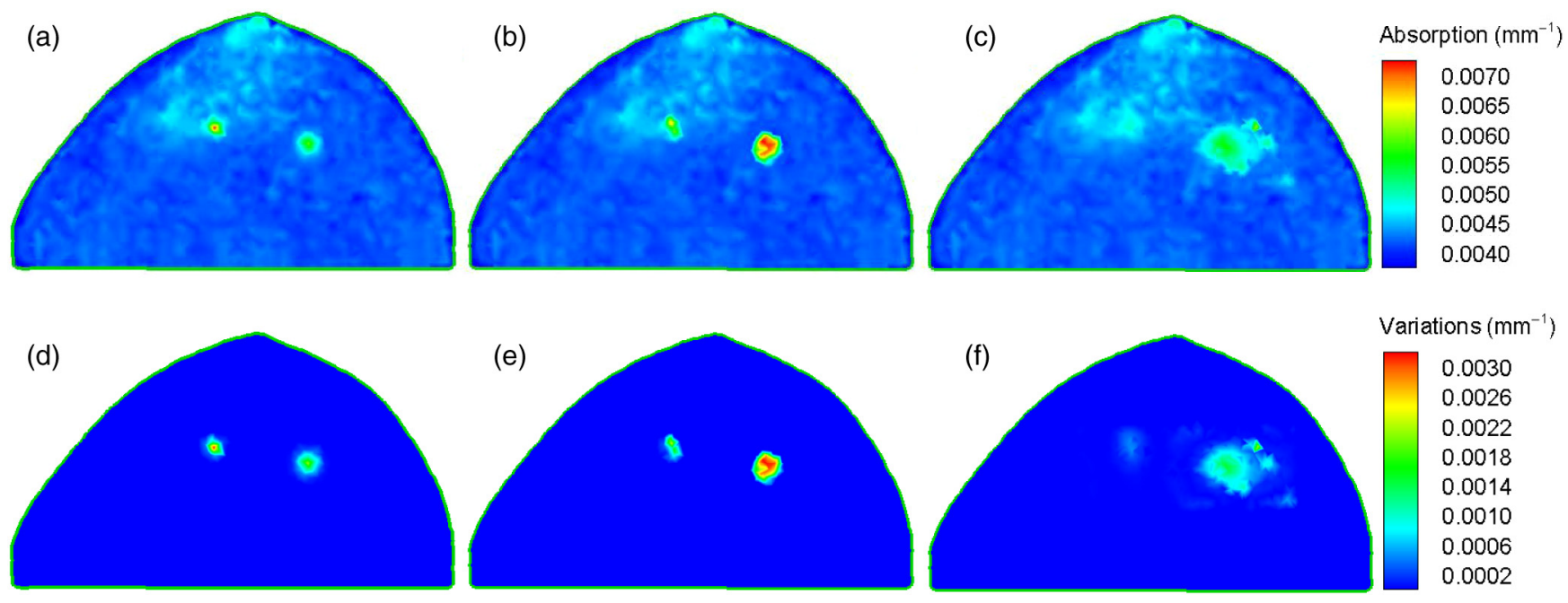

Variations $\left(\mathrm{mm}^{-1}\right)$

Fig. 2 Reconstruction of abnormal tissues from the dataset with $30 \mathrm{~dB}$ noise. (a) The true absorption slice of the phantom at depth of $15 \mathrm{~mm}$, (b) the corresponding reconstructed absorption slice using the proposed method, (c) the corresponding slice reconstructed using the CS-based regularization method, (d) the true variations of abnormalities at depth of $15 \mathrm{~mm}$, (e) the corresponding variations reconstructed using the proposed method, and $(f)$ the variations reconstructed using the CS-based regularization method. 

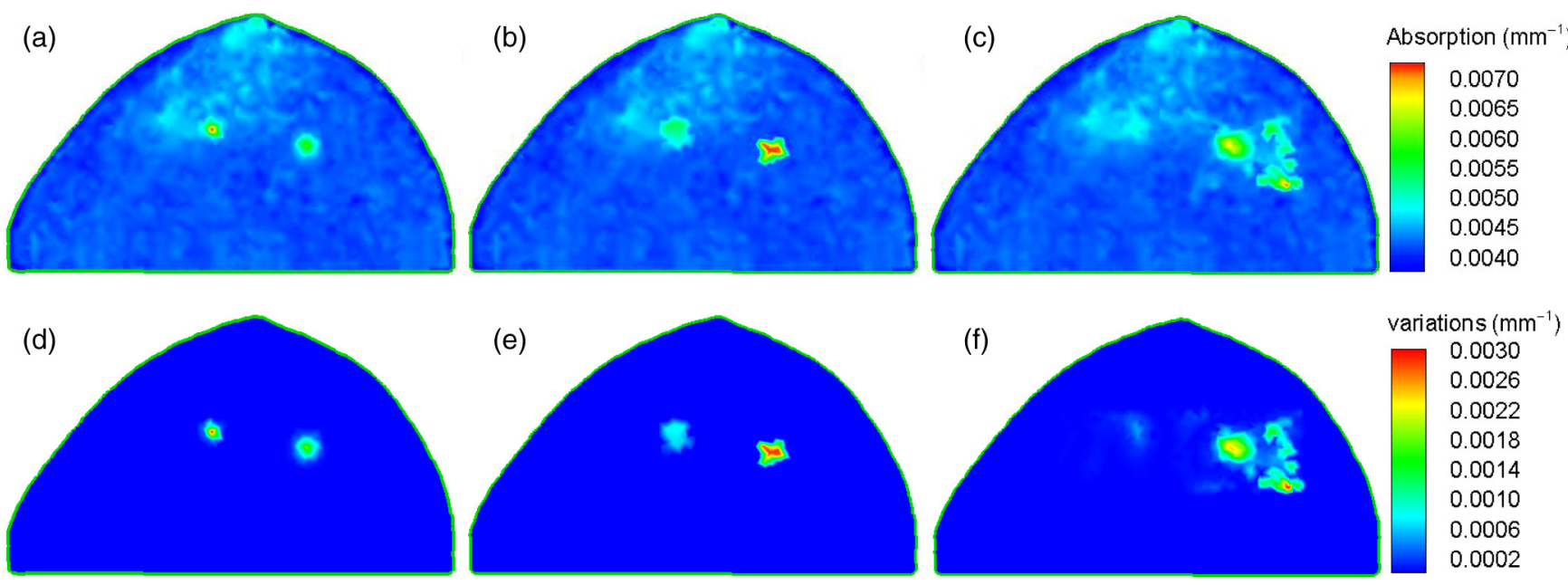

Fig. 3 Reconstruction of abnormal tissues from the dataset with $25 \mathrm{~dB}$ noise. (a) The true absorption slice of the phantom at depth of $15 \mathrm{~mm}$, (b) the corresponding reconstructed absorption slice using the proposed method, (c) the corresponding slice reconstructed using the CS-based regularization method, (d) the true variations of abnormalities at depth of $15 \mathrm{~mm}$, (e) the corresponding variations reconstructed using the proposed method, and ( $\mathrm{f}$ ) the variations reconstructed using the CS-based regularization method.

Table 2 Reconstructed results.

\begin{tabular}{|c|c|c|c|c|c|c|c|c|}
\hline \multirow[b]{2}{*}{$\begin{array}{l}\text { Tumor } \\
\text { type }\end{array}$} & \multirow[b]{2}{*}{$\gamma$} & \multirow[b]{2}{*}{$\sigma$} & \multirow[b]{2}{*}{$\begin{array}{c}\text { True tumor } \\
\text { centroids }(\mathrm{mm})\end{array}$} & \multicolumn{2}{|c|}{ Reconstructed positions of tumors (mm) } & \multirow[b]{2}{*}{$\begin{array}{l}\text { Radius of } \\
\text { region } \bar{\Omega}\end{array}$} & \multicolumn{2}{|c|}{$\begin{array}{l}\text { Average relative error of } \\
\text { reconstructed absorption } \\
\text { coefficients of tumors }\end{array}$} \\
\hline & & & & 30-dB noise & 25-dB noise & & $\begin{array}{c}\text { Dataset with } \\
\quad 30 \mathrm{~dB}\end{array}$ & $\begin{array}{c}\text { Dataset } \\
\text { with } 25 \mathrm{~dB}\end{array}$ \\
\hline 1 & 1.2 & 2.3 & $(140,49,15)$ & $(140.18,47.60,15.27)$ & $(142.38,48.92,15.24)$ & $7(\mathrm{~mm})$ & $4.5 \%$ & $6.7 \%$ \\
\hline II & 2 & 3.6 & $(188,41,15)$ & $(188.33,40.52,14.32)$ & $(190.71,41.40,14.02)$ & $8(\mathrm{~mm})$ & $7.2 \%$ & $10.5 \%$ \\
\hline
\end{tabular}

image reconstruction from the same datasets using a compressive sensing (CS)-based regularization method. ${ }^{11,12,14,27}$ These results demonstrate that the proposed method can achieve more accurate and stable reconstructions than the state-of-theart CS-based regularization reconstruction method.

\section{Discussions and Conclusion}

We have proposed a new optical tomographic imaging method for the detection of breast cancer. This method splits the image reconstruction problem into the localization of abnormal tissues and quantification of absorption variations. The localization of abnormal tissues is performed based on a new well-posed optimization model, which can be solved via DE optimization method to achieve a stable reconstruction. For the early stage cancer detection, it is neither correct nor efficient to assume too many tumors. As far as the detection of a few numbers of tumors in a breast is concerned, the proposed image reconstruction method is very efficient to solve the optimization model of Eq. (10) for the determination of tumor central positions. Around the determined positions of tumors, the quantification of abnormal absorption variations is then determined in localized regions of relatively small extents. Consequently, the number of unknown variables can be greatly reduced to overcome the underdetermined nature of DOT, allowing for accurate and stable reconstruction of the abnormal absorption variations in the breast. The numerical simulations based on the realistic digital breast phantom have been performed to verify the feasibility of this method. The proposed method provides a practical and noninvasive tool for enhancing the accuracy of tumor diagnostics.

Our numerical simulations are based on CW excitation, which provide high signal-to-noise ratio of measured photon fluence rate. The proposed method can be directly extended to time-resolved/frequency-domain optical tomography, which provides more measurement information to enhance accuracy and stability of image reconstruction. In addition, this proposed reconstruction method is based on the diffuse approximation model for high computational efficiency, which works well in breast tissues with weak absorbing and high scattering. It is also straightforward to extend our method to more accurate photon transport models, such as RTE, ${ }^{15}$ phase approximation, ${ }^{16}$ or Monte Carlo simulation. ${ }^{28}$ At the same time, the graphic processing unit can be used to achieve high-performance computing to accelerate the computation of the photon transport models.

\section{Disclosures}

No conflicts of interest, financial or otherwise, are declared by the authors. 


\section{Acknowledgments}

This work was supported by the National Institutes of Health Grant NIH/NIBIB R01 EB019443.

\section{References}

1. M. Herranz and A. Ruibal, "Optical imaging in breast cancer diagnosis: the next evolution," J. Oncol. 2012, 1-10 (2012).

2. R. J. Bleicher and M. Morrow, "MRI and breast cancer: role in detection, diagnosis, and staging," Oncology (Williston Park) 21(12), 15211528 (2007).

3. N. Wasif et al., "MRI versus ultrasonography and mammography for preoperative assessment of breast cancer," Am. Surg. 75(10), 970975 (2009).

4. A. Godavarty et al., "Diagnostic imaging of breast cancer using fluorescence-enhanced optical tomography: phantom studies," J. Biomed. Opt. 9(3), 488-496 (2004).

5. D. Asanuma et al., "Sensitive beta-galactosidase-targeting fluorescence probe for visualizing small peritoneal metastatic tumours in vivo," Nat. Cоттии. 6, 6463 (2015).

6. S. R. Arridge, "Methods in diffuse optical imaging," Philos. Trans. R. Soc. A 369(1955), 4558-4576 (2011).

7. H. Dehghani, "Numerical modelling and image reconstruction in diffuse optical tomography," Philos. Trans. R. Soc. A 367(1900), 3073-3093 (2009).

8. Q. Zhu et al., "Benign versus malignant breast masses: optical differentiation with US-guided optical imaging reconstruction," Radiology 237(1), 57-66 (2005).

9. V. Quaresima, S. J. Matcher, and M. Ferrari, "Identification and quantification of intrinsic optical contrast for near-infrared mammography," Photochem. Photobiol. 67(1), 4-14 (1998).

10. M. A. O'Leary et al., "Experimental images of heterogeneous turbid media by frequency-domain diffusing-photon tomography," Opt. Lett. 20(5), 426-428 (1995).

11. M. A. Ansari et al., "Diffuse optical tomography: image reconstruction and verification," J. Lasers Med. Sci. 5(1), 13-18 (2014).

12. O. Lee et al., "Compressive diffuse optical tomography: noniterative exact reconstruction using joint sparsity," IEEE Trans. Med. Imaging 30(5), 1129-1142 (2011).

13. $\mathrm{L}$. Zhao et al., " $\mathrm{L}_{\mathrm{p}}$ regularization for early gate fluorescence molecular tomography," Opt. Lett. 39(14), 4156-4159 (2014).

14. M. Suzen, A. Giannoula, and T. Durduran, "Compressed sensing in diffuse optical tomography," Opt. Express 18(23), 23676-23690 (2010).

15. S. R. Arridge, "Optical tomography in medical imaging," Inverse Probl. 15, R41-R93 (1999).

16. W. Cong et al., "Modeling photon propagation in biological tissues using a generalized Delta-Eddington phase function," Phys. Rev. E 76(5), 051913 (2007).

17. M. Schweiger et al., "The finite-element method for the propagation of light in scattering media: boundary and source conditions," Med. Phys. 22(11), 1779-1792 (1995).
18. http://www.nextengine.com

19. W. Cong et al., "Practical reconstruction method for bioluminescence tomography," Opt. Express 13(18), 6756-6771 (2005).

20. C. C. Paige and M. A. Saunders, "LSQR: an algorithm for sparse linear equations and sparse least squares," ACM Trans. Math. Software 8, 4371 (1982)

21. B. Deng et al., "Characterization of structural-prior guided optical tomography using realistic breast models derived from dual-energy x-ray mammography," Biomed. Opt. Express 6(7), 2366-2379 (2015).

22. http://mcx.sourceforge.net/cgi-bin/index.cgi?DigiBreast

23. S. L. Jacques, "Optical properties of biological tissues: a review," Phys. Med. Biol. 58(11), R37-R61 (2013).

24. V. G. Peters et al., "Optical properties of normal and diseased human breast tissues in the visible and near infrared," Phys. Med. Biol. 35(9), 1317-1334 (1990).

25. T. Durduran et al., "Bulk optical properties of healthy female breast tissue," Phys. Med. Biol. 47(16), 2847-2861 (2002).

26. H. Key et al., "Optical attenuation characteristics of breast tissues at visible and near-infrared wavelengths," Phys. Med. Biol. 36(5), 579590 (1991).

27. C. B. Shaw and P. K. Yalavarthy, "Performance evaluation of typical approximation algorithms for nonconvex $1_{\mathrm{p}}$-minimization in diffuse optical tomography," J. Opt. Soc. Am. A 31(4), 852-862 (2014).

28. J. Chen, Q. Fang, and X. Intes, "Mesh-based Monte Carlo method in time-domain widefield fluorescence molecular tomography," J. Biomed. Opt. 17(10), 106009 (2012)

Wenxiang Cong is a research scientist in the Department of Biomedical Engineering, Rensselaer Polytechnic Institute. His research interests include x-ray computed tomography, optical molecular tomography, and $\mathrm{x}$-ray phase imaging.

Xavier Intes is a professor in the Department of Biomedical Engineering, Rensselaer Polytechnic Institute, and an AIMBE fellow. He obtained his PhD from the Université de Bretagne Occidentale and postdoctoral training at the University of Pennsylvania. He acted as the chief scientist of Advanced Research Technologies Inc. His research interests are on the application of diffuse functional and molecular optical techniques for biomedical imaging in preclinical and clinical settings.

Ge Wang is chair professor and director of Biomedical Imaging Center, Rensselaer Polytechnic Institute, USA. He authored the first paper on spiral cone-beam/multislice CT and the first paper on bioluminescence tomography. His group published the first papers on interior tomography and omni-tomography. His results were featured in Nature, Science, and PNAS. His interest includes x-ray $\mathrm{CT}$, optical molecular tomography, multimodality imaging, and machine learning. He is a fellow of SPIE, OSA, AIMBE, AAPM, and AAAS. 